\title{
Lactate on emergency department arrival as a predictor of mortality and site-of-care in pneumonia patients: a cohort study
}

\author{
Yun-Xia Chen, Chun-Sheng Li
}

Emergency Department of Beijing Chao-Yang Hospital, Affiliated to Capital Medical University, Beijing, China

\section{Correspondence to} Professor Chun-Sheng Li, Emergency Department of Beijing Chao-Yang Hospital Affiliated to Capital Medical University, Chaoyang District, No. 8, Sorth Road of Worker's Stadium, Chaoyang District, Beijing 100020, China; Icscyyy@wo.cn.

Received 21 October 2014 Revised 30 January 2015 Accepted 6 February 2015 Published Online First 23 February 2015

CrossMark

To cite: Chen Y-X, Li C-S. Thorax 2015;70:404-410.

\begin{abstract}
Objective To investigate the predictive performance of lactate, CURB-65, and a combination of lactate and CURB-65 (LAC-CURB-65) for mortality, hospitalisation and intensive care unit (ICU) admission in pneumonia patients in the emergency department (ED).

Methods Consecutive adult patients with pneumonia presenting from January 2012 to May 2014 were divided into low-, moderate- and high-risk groups according to lactate $(<2.0,2.0-4.0,>4.0 \mathrm{mmol} / \mathrm{L})$, CURB-65 ( $\leq 1,2, \geq 3)$ and LAC-CURB-65 (patients with two low risks, any moderate risk, any high risk) values. Mortality, hospitalisation and ICU admission rates were compared between risk classes.

Results Of 1641 patients, 861 (53\%) were hospitalised (38\% to a general ward, 15\% to the ICU) while the remaining $780(47 \%)$ were treated as outpatients or observed in the ED. 547/1641 (33\%) patients died within 28 days. Lactate and CURB-65 were higher in patients who died, were hospitalised or were admitted to the ICU compared with patients who were

\section{Key messages}

What is the key question?

- How do lactate, CURB-65 and both combined (LAC-CURB-65) perform in predicting mortality, hospitalisation and intensive care unit (ICU) admission in pneumonia patients who visited our emergency department (ED)?

\section{What is the bottom line?}

- Lactate is superior to CURB-65 in predicting mortality and determining site-of-care for pneumonia patients in the ED.

\section{Why read on?}

- As a new risk classification system, LAC-CURB-65 provided superior performance in predicting mortality, hospitalisation and ICU admission in pneumonia patients in the ED.
\end{abstract} not $(p<0.001)$. Lactate and CURB-65 independently predicted outcomes. The performance of lactate in predicting 28-day mortality, hospitalisation and ICU admission was higher than that of CURB-65 $(p<0.01)$. For LAC-CURB-65, patients at low or moderate risk had mortality rates of $2 \%$ and $14 \%$, respectively, and hospitalisation rates of $15 \%$ and $40 \%$, respectively, while none were admitted to ICU. Patients at high risk had the highest mortality $(52 \%)$, hospitalisation $(70 \%)$ and ICU admission rates (27\%).

Conclusions Lactate is superior to CURB-65 in predicting mortality, hospitalisation and ICU admission in pneumonia patients in the ED. LAC-CURB-65 significantly improved the predictive value of CURB-65.

\section{INTRODUCTION}

Pneumonia is the leading cause of sepsis, which can progress to severe sepsis, septic shock and death. Pneumonia independently predicts 28 -day in-hospital mortality in septic patients admitted to the intensive care unit (ICU). ${ }^{1}$ A previous study showed that pneumonia was the primary cause of unplanned ICU transfer within $24 \mathrm{~h}$ of ward arrival from the emergency department (ED). The mortality rate for unplanned ICU transfer patients was higher than that of direct ICU admissions. ${ }^{2}$ Another study found that late but not early (on the same day as ED presentation) admission to the ICU is associated with a higher 28-day mortality rate for patients hospitalised with community-acquired pneumonia (CAP). ${ }^{3}$ Timely and effective initial assessment, decisions on care setting, and management are crucial to improving outcomes.

The CURB- $65^{4}$ (Confusion, Urea $>7 \mathrm{mmol} / \mathrm{L}$, Respiratory rate $\geq 30 / \mathrm{min}$, Blood pressure $<90 \mathrm{~mm} \mathrm{Hg}$ systolic and/or $\leq 60 \mathrm{~mm} \mathrm{Hg}$ diastolic, and age $\geq 65$ years) score has been widely used in CAP patients. It was primarily designed to predict mortality and identify low-risk patients potentially suitable for ambulatory management. The simplicity of calculation made it superior to other more complex score systems used in a crowded ED. A meta-analysis including 23 was effective for identifying patients with CAP at low risk of death. ${ }^{5}$ However, for most high-risk patients sent to an ED, including those with CAP and healthcare-associated pneumonia (HCAP) whose mortality rate was higher, ${ }^{67}$ identification of the highrisk cohort was of more importance. Another metaanalysis reported that the area under the ROC (receiver operating characteristic) curve (AUC) of CURB-65 in predicting ICU admission was $0.69 .{ }^{8}$ CURB-65 is a robust prognostic indicator, but has been recommended for use in conjunction with clinical judgement. ${ }^{9}$ Additionally, CURB-65 was derived and validated in CAP patients. Thus, its predictive performance for mortality and site-of-care was not clear in patients with HCAP or unselected pneumonia.

Lactate has been used for many years in the assessment of perfusion status and as a measure of studies in 22753 participants found that CURB-65 
illness. Elevated lactate was shown to be independently associated with mortality rate in critically ill patients. ${ }^{10}$ Even intermediate initial serum lactate was an indicator of mortality independent of clinically apparent organ dysfunction and shock in severe sepsis patients in the ED. ${ }^{11}$ In hospitalised patients, increased lactate indicated high mortality, mechanical ventilation, vasopressor requirement, and a high incidence of ICU admission. ${ }^{11-13}$ Lactate performed well in risk assessment studies in septic patients; the cut-off values ( 2 and $4 \mathrm{mmol} / \mathrm{L}$ ) were quite similar in different reports. ${ }^{12}{ }^{14} 15$ Even in children with pneumonia, the risk classification performance of lactate was still reasonably accurate. ${ }^{16}$ Previous studies have proved the usefulness of lactate in prognosis and risk stratification, but few have investigated its performance in site-of-care decisions in adult pneumonia patients in the ED.

The present study aimed to investigate the prognostic value, and more importantly, the site-of-care decision-making performance of lactate, CURB-65 and a combination of lactate and CURB-65 (LAC-CURB-65) in unselected pneumonia patients in the ED.

\section{PATIENTS AND METHODS}

\section{Setting and design of the study}

This was a prospective observational clinical study carried out in the ED of Beijing Chao-Yang Hospital, a teaching hospital of Capital Medical University with approximately 250000 ED visits per year. The study was approved by the Beijing Chao-Yang Hospital ethics committee, and written informed consent was obtained from patients or their relatives. Enrolled patients were managed according to international and local guidelines for the management of adults with pneumonia. ${ }^{69} 17$ Hospitalisation decisions were made by physicians who were blinded to the study. Patients with high CURB-65 values, more comorbidities, and a tendency toward deterioration were hospitalised. Haemodynamically unstable patients who required vasopressors, haemodynamic monitoring, and organ replacement therapy (invasive mechanical ventilation and/or continuous renal replacement therapy) were admitted to the ICU.

\section{Study cohort}

Consecutive patients who visited the ED with suspected pneumonia were screened from January 2012 to May 2014. The inclusion criteria were age $\geq 18$ years, new infiltrates on chest radiograph, and two or more symptoms consistent with pneumonia including cough, dyspnoea, fever, sputum production, breathlessness and/or pleuritic chest pain. Patients with pulmonary embolism (PE) or oedema on CT pulmonary angiogram were excluded. Patients positive for HIV or chronically immunosuppressed (including those with solid organ transplants, splenectomy or active tumour, or receiving corticosteroids or other immunosuppressive agents) were also excluded. The enrolled patients included participants with CAP and HCAP. $^{6}$

\section{Data collection}

General information on enrolled patients collected on ED arrival included demographic characteristics, telephone number, medical ID, comorbid conditions, vital signs, results of laboratory tests and imaging examinations. Arterial lactate concentration was measured using a Stat Profile pHOx analyser (Nova Biomedical, Waltham, Massachusetts, USA) within $1 \mathrm{~h}$ after ED arrival. The CURB-65 score was calculated in every patient using data collected on ED arrival.

\section{Definition of comorbidities}

Chronic obstructive pulmonary disease (COPD) was defined as a diagnosis of COPD in the past. Cardiovascular disease was defined as coronary artery disease (angina or old myocardial infarction) and/or congestive heart failure (any New York Heart Association class). Stroke was defined as ischaemic and/or haemorrhagic. Tumour was defined as a neoplasm of any type. Renal disease was mainly defined as chronic renal failure and included patients on dialysis. Liver disease referred to cirrhosis of any severity. Diabetes mellitus included both insulin-dependent and non-insulin-dependent disease.

\section{Outcome variables}

All patients were followed up for to 28 days through medical records or by telephone, and 28-day all-cause mortality was the primary outcome. The secondary outcomes included hospitalisation (admission to a general ward or the ICU) and ICU admission.

\section{Statistical analysis}

All data were analysed by SPSS V.16.0 (SPSS, Chicago, Illinois, USA). Data with a skewed distribution were expressed as medians and quartiles and were analysed by the Mann-Whitney U test. The $\chi^{2}$ test was used for comparison of frequencies. Any variable (comorbidities, important laboratory and imaging results) with a $\mathrm{p}$ value of $<0.05$ between patients with different outcomes (nonsurvivors and survivors, hospitalised and non-hospitalised, ICU and non-ICU admission, respectively) was eligible for inclusion in a binary logistic regression, which identified the independent predictors of outcomes at a level of $\mathrm{p}<0.05$. Next, a stepwise binary logistic regression was performed, with a separate model for each outcome (mortality, hospitalised and ICU admission), to yield three final models of significant predictors. As vital signs are included in CURB-65, they were not analysed separately again. ROC curves were constructed and the AUCs were determined to assess predictive value. For comparison of the AUCs, $\mathrm{Z}=\left(\mathrm{A}_{1}-\mathrm{A}_{2}\right) / \sqrt{\mathrm{SE}_{1}^{2}+\mathrm{SE}_{2}^{2}}$ was used, the test values being $\mathrm{Z}_{0.05}=1.96$ and $\mathrm{Z}_{0.01}=2.58$. Based on thresholds determined according to ROC curve analysis, prognostic parameters including positive and negative predictive values and positive and negative likelihood ratios were also calculated. Lactate and CURB-65 were defined as two independent risk factors with each containing three risk classes: low-, moderate- and high-risk. The CURB-65 risk category thresholds matched those proposed in the original study: low- (CURB-65 $\leq 1)$, moderate-(CURB-65 =2) and high-risk (CURB-65 $\geq 3$ ). ${ }^{4}$ Lactate risk classes were defined as follows: low risk (lactate $<2 \mathrm{mmol} / \mathrm{L})$, moderate risk $(2-4 \mathrm{mmol} / \mathrm{L})$ and high risk $(>4 \mathrm{mmol} / \mathrm{L})$. The lactate thresholds were determined from studies in septic patients. ${ }^{11} 12141518$ The cohort was then separated into three risk groups according to the combination of lactate and CURB-65 (LAC-CURB-65): patients with two low risks, patients with any moderate risk, and those with a high risk. The 28-day mortality, hospitalisation and ICU admission were compared among the three groups. All statistical tests were twotailed, and $\mathrm{p}<0.05$ was considered statistically significant.

\section{RESULTS}

\section{Characteristics of the study cohort}

A total of 1769 patients were evaluated at recruitment, and 104 were excluded because the final diagnosis was not pneumonia. Twenty-four patients were lost to follow-up. The study ultimately enrolled 1641 pneumonia patients. The baseline characteristics of patients are listed in table 1 . The median time to admission to ICU was 1 (range $1-2$ ) day (1 day indicated the 
Table 1 Baseline characteristics of the study cohort

\begin{tabular}{|c|c|c|c|c|c|c|c|c|c|c|}
\hline & Entire cohort & Non-survivors & Survivors & $\mathrm{p}$ Value & Hospitalised & Non-hospitalised & $\mathrm{p}$ Value & ICU admission & Non-ICU admission & $p$ Value \\
\hline $\mathrm{N}$ & 1641 & 547 & 1094 & & 861 & 780 & & 246 & 1395 & \\
\hline Age (years) & $73(62-79)$ & $75(66-81)$ & $73(60-78)$ & $<0.001$ & $74(65-80)$ & $72(59-78)$ & $<0.001$ & $75(68-81)$ & $73(61-79)$ & $<0.001$ \\
\hline Male (\%) & 59 & 58 & 59 & 0.48 & 57 & 61 & 0.12 & 57 & 59 & 0.61 \\
\hline \multicolumn{11}{|l|}{ Comorbidities } \\
\hline COPD $(\%)$ & 34 & 36 & 32 & 0.10 & 36 & 31 & 0.01 & 43 & 32 & 0.001 \\
\hline CVD (\%) & 28 & 30 & 26 & 0.07 & 30 & 26 & 0.13 & 30 & 27 & 0.33 \\
\hline Stroke $(\%)$ & 12 & 14 & 11 & 0.08 & 12 & 12 & 0.79 & 13 & 12 & 0.66 \\
\hline Tumour (\%) & 9 & 11 & 8 & 0.04 & 10 & 8 & 0.16 & 11 & 9 & 0.32 \\
\hline Renal (\%) & 5 & 6 & 4 & 0.08 & 6 & 4 & 0.22 & 7 & 5 & 0.26 \\
\hline Liver (\%) & 8 & 9 & 8 & 0.25 & 8 & 8 & 0.62 & 10 & 8 & 0.19 \\
\hline DM (\%) & 15 & 13 & 16 & 0.13 & 17 & 14 & 0.06 & 17 & 15 & 0.38 \\
\hline \multicolumn{11}{|l|}{ Vital signs } \\
\hline $\operatorname{MAP}(\mathrm{mm} \mathrm{Hg})$ & $80(67-93)$ & $70(54-83)$ & $84(73-97)$ & $<0.001$ & $77(60-90)$ & 83 (71-97) & $<0.001$ & $67(50-82)$ & $82(70-95)$ & $<0.001$ \\
\hline Respiratory rate (bpm) & $27(24-32)$ & $28(24-32)$ & $26(24-32)$ & 0.01 & $28(24-32)$ & $26(24-32)$ & 0.09 & $30(24-32)$ & $26(24-32)$ & 0.003 \\
\hline Temperature $\left({ }^{\circ} \mathrm{C}\right)$ & $36.8(36.2-38.2)$ & $36.8(36.2-38.2)$ & $36.8(36.2-38.1)$ & 0.83 & $36.8(36.2-38.2)$ & $36.8(36.2-38.1)$ & 0.65 & $37(36.2-38.2)$ & $36.8(36.2-38.1)$ & 0.62 \\
\hline Heart rate (bpm) & $110(98-126)$ & 110 (99-126) & $110(97-125)$ & 0.20 & $110(98-128)$ & $110(98-124)$ & 0.22 & $110(98-126)$ & $110(98-126)$ & 0.99 \\
\hline Mental confusion (\%) & 28 & 46 & 20 & $<0.001$ & 37 & 19 & $<0.001$ & 66 & 22 & $<0.001$ \\
\hline \multicolumn{11}{|l|}{ Laboratory results } \\
\hline WBC $\left(\times 10^{9} / \mathrm{L}\right)$ & $11.2(7.7-15.6)$ & $11.0(7.6-15.1)$ & $11.2(7.8-15.6)$ & 0.48 & $11.2(7.7-15.5)$ & $11.3(7.7-15.7)$ & 0.75 & $11.4(7.6-16)$ & $11.2(7.7-15.5)$ & 0.71 \\
\hline PCT (ng/mL) & $1.1(0.9-3.4)$ & $1.3(0.9-9.4)$ & $1.1(0.9-3.4)$ & $<0.001$ & $1.1(0.9-3.4)$ & $1.1(0.9-3.4)$ & 0.50 & $1.2(0.9-3.7)$ & $1.1(0.9-3.4)$ & 0.15 \\
\hline $\mathrm{PaO}_{2}(\mathrm{~mm} \mathrm{Hg})$ & 75 (59-94) & $71(57-89)$ & $77(60-96)$ & $<0.001$ & $74(59-92)$ & $76(59-96)$ & 0.28 & $69(55-86)$ & $77(60-95)$ & $<0.001$ \\
\hline Platelets $\left(\times 10^{9} / L\right)$ & $184(135-243)$ & $185(136-243)$ & $183(134-244)$ & 0.91 & $186(136-243)$ & $183(134-243)$ & 0.81 & $188(142-246)$ & $183(134-242)$ & 0.73 \\
\hline Creatinine $(\mu \mathrm{mol} / \mathrm{L})$ & $100(75-148)$ & $106(78-170)$ & $98(74-136)$ & $<0.001$ & $102(77-157)$ & $98(73-136)$ & 0.02 & $134(98-228)$ & 96 (72-134) & $<0.001$ \\
\hline Bilirubin $(\mu \mathrm{mol} / \mathrm{L})$ & $12.3(8.3-18.6)$ & $13.1(8.1-19.3)$ & $12.0(8.1-18.3)$ & 0.04 & $12.2(8.2-19)$ & $12.5(8.3-18.3)$ & 0.94 & $12.2(8.2-18.7)$ & $12.3(8.3-18.5)$ & 0.63 \\
\hline Positive sputum & $378 / 1132(33 \%)$ & 143/394 (36\%) & 235/738 (32\%) & 0.13 & 209/583 (36\%) & 169/549 (31\%) & 0.07 & 73/187 (39\%) & 305/945 (32\%) & 0.07 \\
\hline Lactate (mmol/L) & $\begin{array}{l}2.6(1.0-5.1) \\
3.6 \pm 3.4\end{array}$ & $\begin{array}{l}5.4(3.1-8.1) \\
6.1 \pm 4.0\end{array}$ & $\begin{array}{l}1.6(0.8-3.3) \\
2.3 \pm 2.0\end{array}$ & $<0.001$ & $\begin{array}{l}4.4(2.5-7.0) \\
5.1 \pm 3.7\end{array}$ & $\begin{array}{l}1.2(0.6-2.5) \\
1.8 \pm 1.8\end{array}$ & $<0.001$ & $\begin{array}{l}8.5(6.6-11.7) \\
9.0 \pm 4.0\end{array}$ & $\begin{array}{l}2.1(0.9-4.0) \\
2.6 \pm 2.1\end{array}$ & $<0.001$ \\
\hline Multilobar opacities (\%) & 40 & 47 & 37 & $<0.001$ & 42 & 38 & 0.07 & 53 & 38 & $<0.001$ \\
\hline CURB-65 & $2(1-3)$ & $3(2-3)$ & $2(1-3)$ & $<0.001$ & $2(2-3)$ & $2(1-3)$ & $<0.001$ & $3(2-4)$ & $2(1-3)$ & $<0.001$ \\
\hline 28-Day mortality (\%) & 33 & - & - & - & 54 & 11 & $<0.001$ & 85 & 24 & $<0.001$ \\
\hline
\end{tabular}

Skewed distributed data are expressed as medians and quartiles.

COPD, chronic obstructive pulmonary disease; CVD, cardiovascular disease; DM, diabetes mellitus; ICU, intensive care unit; MAP, mean arterial pressure; PaO 2 , arterial oxygen pressure; $\mathrm{PCT}$, procalcitonin; WBC, white blood cells. 
day of ED arrival). The median length of admission to a general ward was 4 (range 2-5) days.

\section{Lactate and CURB-65 levels}

Lactate and CURB-65 were significantly higher in non-survivors than in survivors, in hospitalised than in non-hospitalised patients, and in ICU than in non-ICU admission patients $(\mathrm{p}<0.001)$ (table 1).

Although lactate increased in line with CURB-65 values, the differences between groups were not statistically significant. However, lactate level significantly differed between CURB-65 risk classes (table 2).

\section{Independent predictors of mortality and site-of-care}

Lactate and CURB-65 were independent predictors of mortality, hospitalisation and ICU admission (table 3).

\section{Performance of lactate and CURB-65 in risk classification and site-of-care decisions}

The ROC curves for lactate and CURB-65 are shown in figure 1. The AUCs for lactate in predicting all three outcomes were much larger than those of CURB-65. LAC-CURB-65 significantly improved the predictive value of CURB-65, but not of lactate, for mortality and hospitalisation. LAC-CURB-65 improved the performance of both lactate and CURB-65 in predicting ICU admission. The cut-off values are listed in table 4 .

The 28-day mortality and hospitalisation increased in line with lactate risk class (figure 2). LAC-CURB-65 further highlighted the differences in mortality, hospitalisation and ICU admission between the different risk classes (figure 2C).

\section{Distribution of risk classes of lactate and CURB-65 in patients with different outcomes}

As shown in figure 3, the proportion of high-risk patients by lactate was much higher in non-survivors than in survivors, in hospitalised than in non-hospitalised patients, and in ICU admission than in non-ICU admission patients. A similar trend was found regarding CURB-65 risk classes, but the difference was not as significant. LAC-CURB-65 further increased the proportion of high-risk patients who were non-survivors, hospitalised or ICU admitted, especially in patients admitted to ICU, where all patients were high risk.

\section{DISCUSSION}

The present study revealed that lactate is a better predictor than CURB-65 of mortality, hospitalisation and ICU admission in pneumonia patients in the ED. LAC-CURB-65 provided the best predictive performance.

Table 2 Lactate levels according to CURB-65

\begin{tabular}{llrl}
\hline CURB-65 & Lactate (mmol/L) & CURB-65 & Lactate (mmol/L) \\
\hline $0(n=95)$ & $1.5(0.5-4.2)$ & $\leq 1(n=493)$ & $1.8(0.9-4.2)^{*, * *}$ \\
$1(n=398)$ & $1.9(0.9-4.2)$ & $2(n=535)$ & $2.4(0.9-4.5)^{* *}$ \\
$2(n=535)$ & $2.4(0.9-4.5)$ & $\geq 3(n=613)$ & $3.6(1.4-6.0)$ \\
$3(n=393)$ & $3.2(1.3-5.8)$ & & \\
$4(n=176)$ & $3.9(1.5-6.3)$ & & \\
$5(n=44)$ & $4.4(3.0-6.6)$ & & \\
\hline
\end{tabular}

Skewed distributed data were expressed as medians and quartiles; compare to CURB-65 $\geq 3$

${ }^{*} \mathrm{p}<0.05$, compared to CURB-65 $=2 ;{ }^{*} \mathrm{p}<0.01$.
Table 3 Independent predictors of outcomes

\begin{tabular}{lllllll}
\hline & & & & \multicolumn{2}{c}{$\mathbf{9 5 \%} \mathrm{Cl}$ for OR } \\
\cline { 5 - 7 } Outcomes & $\begin{array}{l}\text { Independent } \\
\text { predictors }\end{array}$ & $\mathbf{p}$ Value & $\mathbf{O R}$ & $\mathbf{5 \%}$ & $\mathbf{9 5 \%}$ \\
\hline \multirow{2}{*}{ 28-Day mortality } & Lactate (mmol/L) & $<0.001$ & 1.584 & 1.503 & 1.670 \\
& CURB-65 & $<0.001$ & 1.798 & 1.603 & 2.018 \\
Hospitalisation & Lactate (mmol/L) & $<0.001$ & 1.688 & 1.589 & 1.793 \\
& CURB-65 & $<0.001$ & 1.273 & 1.149 & 1.410 \\
ICU admission & Lactate (mmol/L) & $<0.001$ & 2.189 & 1.982 & 2.418 \\
& CURB-65 & $<0.001$ & 2.430 & 2.006 & 2.945
\end{tabular}

The results are adjusted for arterial oxygen pressure, creatinine and multilobar opacities. The odds ratio is the change in odds of transfusion for each increasing unit of lactate or CURB-65.

ICU, intensive care unit.

\section{Lactate at ED arrival as a prognostic indicator in pneumonia patients}

The present study demonstrated that lactate is significantly elevated in non-survivors of pneumonia, and independently predicts 28-day mortality. The AUC of lactate in predicting 28-day mortality was 0.823 , which was more accurate than that of CURB-65 (0.692). Several studies have demonstrated that an elevated lactate at admission is an independent predictor of in-hospital mortality in infectious or septic patients. ${ }^{11}{ }^{18-21}$ Even normal lactate concentrations $(<2.3 \mathrm{mmol} / \mathrm{L})$ can be a useful prognostic indicator in septic shock. ${ }^{20}$ In a study conducted in an infectious cohort in the ED, patients with serum lactate of $4 \mathrm{mmol} / \mathrm{L}$ or greater had a 3 -day mortality rate of $22.4 \%$ compared with $1.5 \%$ in patients with a serum lactate of $2.4 \mathrm{mmol} / \mathrm{L}$ or less. ${ }^{19} \mathrm{~A}$ recent study showed that lactate was a robust predictor of death in children with $\mathrm{CAP}^{16}$ However, studies in adult pneumonia patients are still rare. The result of our study provided some evidence that mortality rate could be predicted by measuring lactate on ED arrival in adult patients with relatively severe pneumonia.

The prognostic ability of lactate was proved independent of organ failure and shock in severe sepsis patients. ${ }^{11}$ Previous studies demonstrated that lactate can be used as an early marker of organ failure and occult shock before any detectable changes occur in a patient's vital signs. ${ }^{22}$ Three of the five variables of CURB-65 are vital signs and are indirect and late indicators of circulation dysfunction. This may be the reason why CURB-65 was not as good in predicting mortality in our study. The prognostic ability of lactate independent of organ dysfunction and shock made lactate an earlier and better indicator of death than CURB-65.

\section{Ability of lactate to classify risk}

Mortality was the most widely used outcome in risk classification. The risk class based on lactate in the present study accurately distinguished those patients at high risk of death from those at moderate or low risk. The 28-day mortality rate was as high as $65 \%$ in the high-risk group and $28 \%$ in the moderate group. The large difference in mortality between the two groups showed that lactate can accurately stratify risk. Similar results were reported in critically ill patients including those with sepsis, trauma and shock. ${ }^{11}{ }^{18-21} 23$ The illness severity of the enrolled cohort in these studies varied widely and in-hospital mortality ranged from $4.9 \%$ to $22.9 \%$. However, the cut-off values of lactate used in these studies were quite similar. The level of lactate was stratified as low $(<2.0 \mathrm{mmol} / \mathrm{L})$, 
A

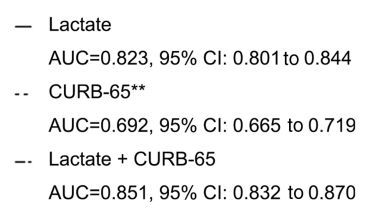

Siginificance $\left({ }^{* *} \mathrm{p}<0.01\right)$ was relative to lactate

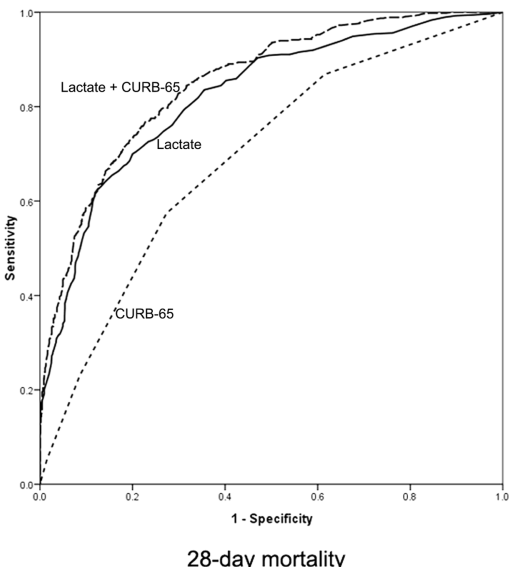

B

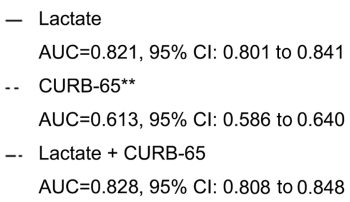

Siginificance $\left({ }^{* *} \mathrm{p}<0.01\right)$ was relative to lactate

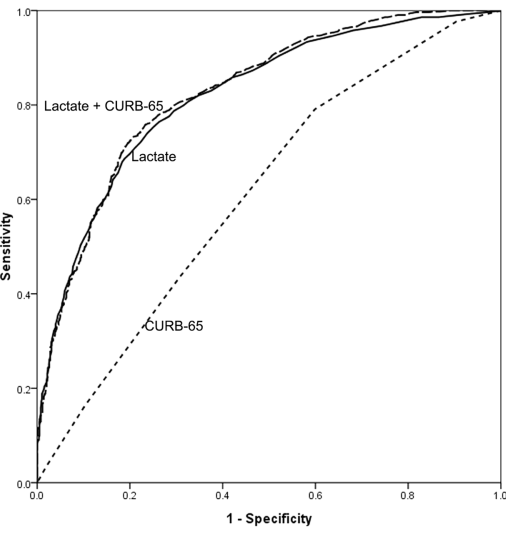

Hospitalisation

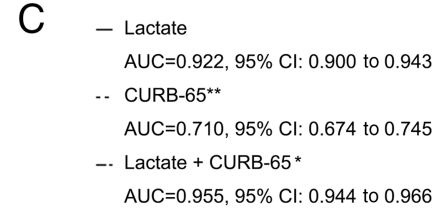

Siginificance $\left({ }^{* *} \mathrm{p}<0.01,{ }^{*} \mathrm{p}<0.05\right)$ was relative to lactate

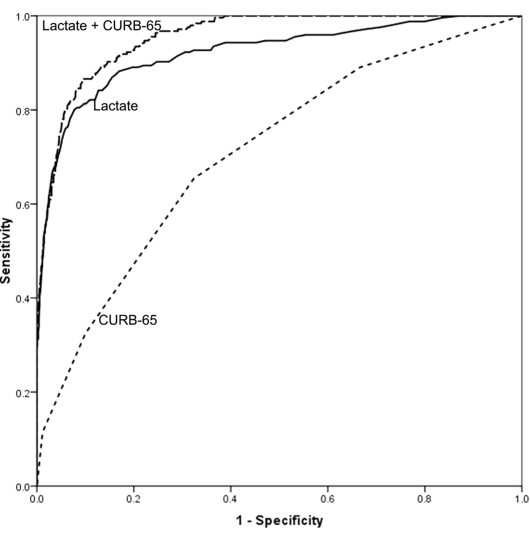

ICU admission

Figure 1 Receiver operating characteristic curves of lactate and CURB-65 in predicting outcomes. (A) 28-Day mortality. (B) Hospitalisation. (C) ICU admission. ${ }^{*} \mathrm{p}<0.05,{ }^{* *} \mathrm{p}<0.01$ vs lactate. ICU, intensive care unit.

intermediate $(2.0-4.0 \mathrm{mmol} / \mathrm{L})$ or high $(>4.0 \mathrm{mmol} / \mathrm{L})$. This stratification performed well in predicting mortality in patients with varying severities of illness. The fact that lactate increased directly with CURB-65 scores in the present study provided further evidence for the ability of lactate to stratify risk. The ability of CURB-65 to stratify risk was also good in our study, but the differences in mortality between risk groups were not as significant as for lactate.

\section{Lactate on ED arrival as an indicator for site-of-care decisions in pneumonia patients}

The performance of lactate in predicting hospitalisation and ICU admission was considerably better than that of CURB-65 in our study. The decision on site-of-care for patients was made on the basis of clinical judgment by ED physicians who were blinded to the study. CURB-65 was one of the assessment variables, while lactate was not routinely used in deciding site-of-care. The results revealed that lactate was more in line with the ED physician's clinical judgment than CURB-65. The AUCs of lactate in predicting hospitalisation and ICU admission were larger than those of CURB-65. The incidence of hospitalisation in our enrolled patients increased directly with lactate risk class, and the differences between groups were significant. However, the performance of CURB-65 in predicting hospitalisation was not as good as that of lactate. Overall, 37\% of patients with a CURB- $65 \leq 1$ were hospitalised. This result shows that CURB-65 did not effectively identify the low-risk patients in the present study. This may be partly owing to the fact that the enrolled cohort was older, had more comorbidities, and had more unstable health that tended to deteriorate after ED arrival. The performance of CURB-65 in predicting ICU admission was similar to that found in other reports. The AUC of CURB-65 in our study was 0.71 , which is similar to the results of two large meta-analyses. ${ }^{58}$

The rate of ICU admission rapidly increased to $39 \%$ in patients with lactate above $4 \mathrm{mmol} / \mathrm{L}$, which was a significantly higher admission rate than in patients with a lower lactate level. This result demonstrated that lactate above $4 \mathrm{mmol} / \mathrm{L}$ is a strong indicator of ICU admission in pneumonia patients in the ED. A previous study revealed that circulating lactate concentrations greater than $4 \mathrm{mmol} / \mathrm{L}$ had a specificity of $98.2 \%$ in predicting the need for hospital admission in patients presenting to the ED. ${ }^{15} \mathrm{~A}$ recent study in suspected infectious paediatric patients in the ED demonstrated that venous lactate was predictive of hospital admission and increased length of stay. ${ }^{24}$ For adult pneumonia patients in the ED, the knowledge of lactate predicting hospitalisation or ICU admission was limited.

Table 4 Predictive performance of independent indicators

\begin{tabular}{|c|c|c|c|c|c|c|c|c|}
\hline Outcomes & Predictors & Cut-off value & Sensitivity (\%) & Specificity (\%) & PPV (\%) & NPV (\%) & LR+ & LR- \\
\hline \multirow[t]{2}{*}{ Mortality } & Lactate (mmol/L) & 4 & 68 & 82 & 65 & 83 & 3.7 & 0.4 \\
\hline & CURB-65 & 2 & 57 & 73 & 51 & 77 & 2.1 & 0.6 \\
\hline \multirow[t]{2}{*}{ Hospitalisation } & Lactate (mmol/L) & 3 & 68 & 82 & 80 & 70 & 3.7 & 0.4 \\
\hline & CURB-65 & 1 & 79 & 40 & 59 & 64 & 1.3 & 0.5 \\
\hline \multirow[t]{2}{*}{ ICU admission } & Lactate (mmol/L) & 6 & 81 & 92 & 63 & 96 & 9.7 & 0.2 \\
\hline & CURB-65 & 3 & 33 & 90 & 36 & 88 & 3.3 & 0.8 \\
\hline
\end{tabular}

ICU, intensive care unit; LR-, negative likelihood ratio; LR+, positive likelihood ratio; NPV, negative predictive value; PPV, positive predictive value. 
A

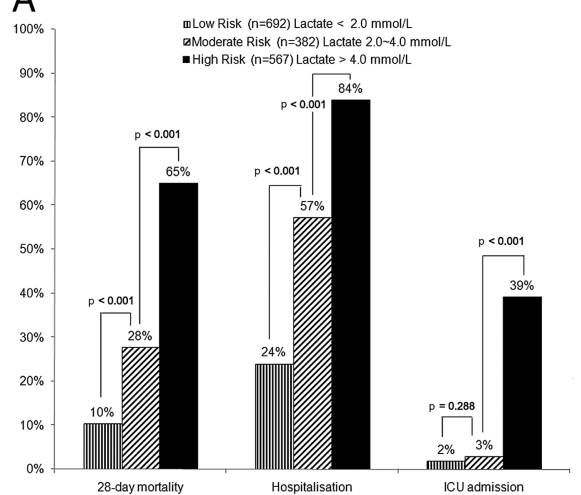

B

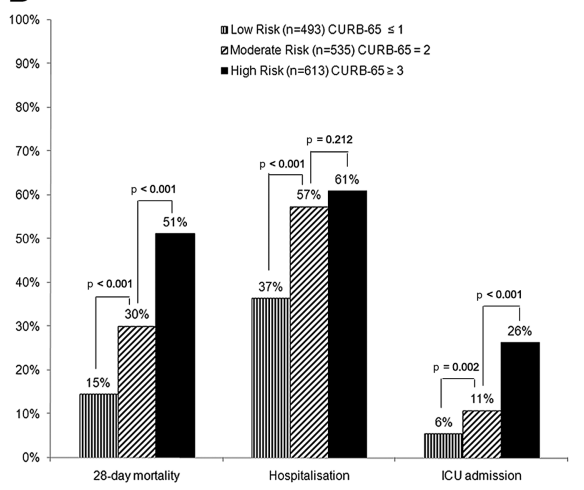

C

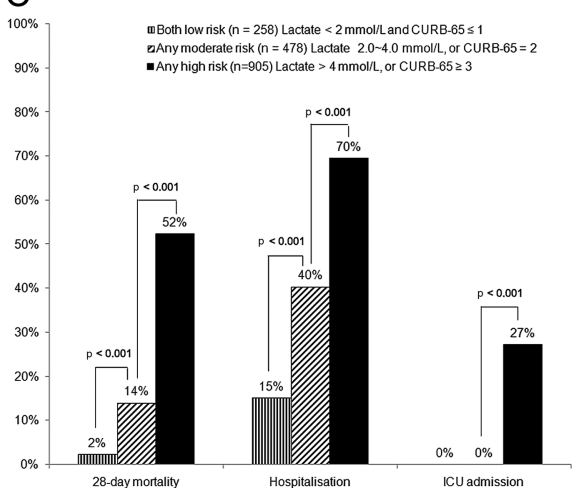

Figure 2 Risk classification of pneumonia patients according to lactate levels and CURB-65. (A) Risk class based on lactate. (B) Risk class based on CURB-65. (C) Risk class based on combined lactate and CURB-65. ICU, intensive care unit.

\section{Combination of lactate and CURB-65 has a better predictive ability than CURB-65 alone}

In the present study, LAC-CURB-65 was significantly better at predicting mortality, hospitalisation and ICU admission. The AUCs of LAC-CURB-65 in predicting all three outcomes were larger than those of CURB-65 alone. For clinical convenience, we did not add lactate to CURB-65 but considered lactate and CURB-65 as two independent risk factors, but also created LAC-CURB-65. This risk classification method effectively distinguished the high-risk patients from low-risk patients. The 28-day mortality, hospitalisation and ICU admission rates were much lower in LAC-CURB-65 low-risk patients than in high-risk patients.

\section{Application of the results}

According the LAC-CURB-65 risk classes, low-risk patients could be observed at home or in the ED for a period, moderate-risk patients should be admitted to a general ward, and high-risk patients should be monitored more closely, treated more aggressively, and admitted to the ICU earlier.

\section{Limitations of the study}

Our study has some limitations. First, this was a single-centre study. Hospitalisation and ICU admission decisions taken on the basis of institutional or departmental guidelines may have played a role in site-of-care decisions. Multicentre prospective studies may decrease this influence.

Second, the high mortality in the study cohort may limit the generalisability of our results to some extent. The reasons for the high mortality were as follows: (1) the advanced age of the study cohort; (2) pneumonia patients with cormobidities tended to also develop multiple organ dysfunction syndrome; (3) as a large tertiary teaching hospital, we received numerous severe pneumonia patients transferred from smaller hospitals and primary healthcare institutions; before being transferred to our $\mathrm{ED}$, these patients were hospitalised, treated with more than one antibiotic, and perhaps intubated and ventilated mechanically, but the intervention was not effective; (4) almost all patients at high risk of death are sent to an ED for initial assessment in our country, while many patients with less severe illness visit specialist clinics directly and are not evaluated in an ED; (5) the patients admitted to ICU were those who had already developed multiple organ dysfunction, were intubated and mechanically ventilated, and needed dynamic monitoring or continuous renal replacement therapy; and (6) bedridden patients with repeated pneumonias and nursing-home resident patients were included. All these factors tended to result in higher mortality.

Third, information on microorganisms was incomplete, as it is difficult to obtain good samples in the ED. The influence of microorganisms on mortality was controversial in different studies. In the present study, the sputum culture was not different between patients with different outcomes.

Fourth, most patients requiring invasive mechanical ventilation, vasopressors or continuous renal replacement therapy were admitted to an ICU in our study, and an accurate record of life support care was not available.

Although our study had these limitations, it confirmed the prognostic and site-of-care deciding value of CURB-65 in a relatively high-risk population. Also, our study demonstrated that
A

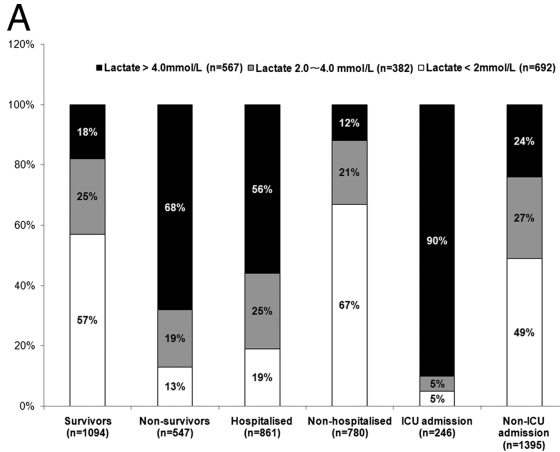

B

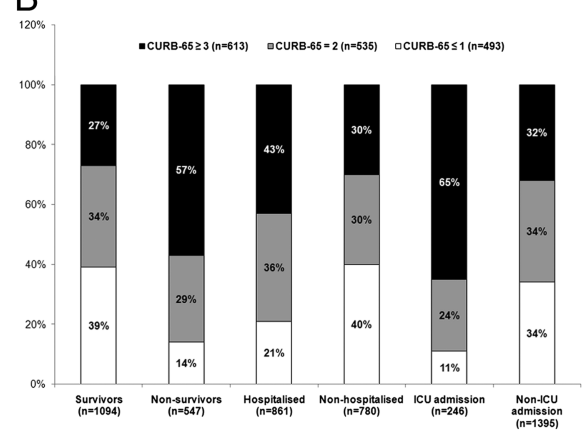

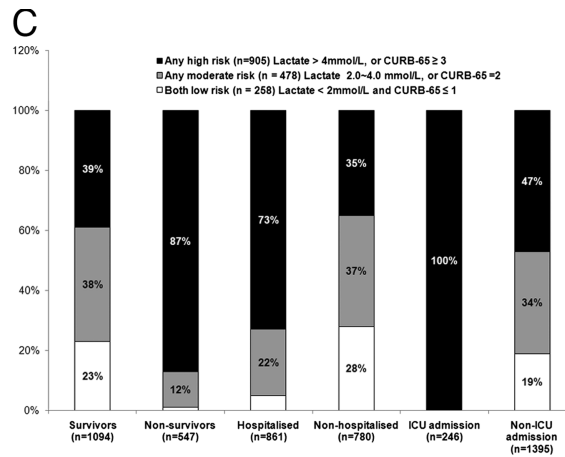

Figure 3 Risk class distribution for lactate, CURB-65 and the combination of lactate and CURB-65. (A) Distribution of lactate risk class in patients with different outcomes. (B) Distribution of CURB-65 risk class in patients with different outcomes. (C) Distribution of combination lactate and CURB-65 risk class in patients with different outcomes. ICU, intensive care unit. 
lactate was superior to CURB-65 and improved the predictive ability of CURB-65 in an unselected pneumonia population different from CAP cohorts.

\section{CONCLUSIONS}

Lactate is a valuable biomarker in predicting mortality and assessing level of care in pneumonia patients in the ED. The LAC-CURB-65 system is much better than CURB-65 alone for prognosis and risk classification.

Acknowledgements The authors sincerely thank Bo Liu, Qin Yin and Yun-Zhou Zhao for their excellent assistance. The authors also thank both the emergency department and the biochemistry laboratory staff for their helpful contributions to the study.

Contributors $\mathrm{Y}-\mathrm{XC}$ conceived and designed the research, acquired, analysed and interpreted the data, drafted the manuscript and revised it critically for important intellectual content. C-SL conceived and designed the research, analysed and interpreted the data, gave final approval of the version to be published, and was responsible for the overall content as guarantor. Both authors read and approved the final manuscript.

Competing interests None.

Ethics approval Received from the Beijing Chao-Yang Hospital ethics committee.

Provenance and peer review Not commissioned; internally peer reviewed.

\section{REFERENCES}

1 Kim WY, Lee YJ, Yeon Lim S, et al. Clinical characteristics and prognosis of pneumonia and sepsis: multicenter study. Minerva Anestesiol 2013;79:1356-65.

2 Delgado MK, Liu V, Pines JM, et al. Risk factors for unplanned transfer to intensive care within 24 hours of admission from the emergency department in an integrated healthcare system. J Hosp Med 2013;8:13-19.

3 Renaud B, Brun-Buisson C, Santin A, et al. Outcomes of early, late, and no admission to the intensive care unit for patients hospitalized with community-acquired pneumonia. Acad Emerg Med 2012;19:294-303.

4 Lim WS, van der Eerden MM, Laing $R$, et al. Defining community acquired pneumonia severity on presentation to hospital: an international derivation and validation study. Thorax 2003;58:377-82.

5 Loke YK, Kwok CS, Niruban A, et al. Value of severity scales in predicting mortality from community-acquired pneumonia: systematic review and meta-analysis. Thorax 2010;65:884-90.

6 American Thoracic Society; Infectious Diseases Society of America. Guidelines for the management of adults with hospital-acquired, ventilator-associated, and healthcare-associated pneumonia. Am J Respir Crit Care Med 2005;171:388-416.
7 Polverino E, Torres A, Menendez R, et al. Microbial aetiology of healthcare associated pneumonia in Spain: a prospective, multicentre, case-control study. Thorax 2013;68:1007-14.

8 Marti C, Garin N, Grosgurin 0, et al. Prediction of severe community-acquired pneumonia: a systematic review and meta-analysis. Crit Care 2012;16:R141.

9 Lim WS, Baudouin SV, George RC, et al. BTS guidelines for the management of community acquired pneumonia in adults: update 2009. Thorax 2009;64(Suppl 3): iii1-55.

10 Khosravani $H$, Shahpori R, Stelfox HT, et al. Occurrence and adverse effect on outcome of hyperlactatemia in the critically ill. Crit Care 2009;13:R90.

11 Mikkelsen ME, Miltiades AN, Gaieski DF, et al. Serum lactate is associated with mortality in severe sepsis independent of organ failure and shock. Crit Care Med 2009;37:1670-7.

12 Tang Y, Choi J, Kim D, et al. Clinical predictors of adverse outcome in severe sepsis patients with lactate 2 to $4 \mathrm{mM}$ admitted to the hospital. QJM. Published Online First: 4 Sep 2014. doi:http://dx.doi.org/10.1093/qjmed/hcu186

13 Azevedo LC, Park M, Salluh JI, et al. Clinical outcomes of patients requiring ventilatory support in Brazilian intensive care units: a multicenter, prospective, cohort study. Crit Care 2013;17:R63.

14 Bakker J, Gris P, Coffernils M, et al. Serial blood lactate levels can predict the development of multiple organ failure following septic shock. Am J Surg 1996;171:221-6.

15 Aduen J, Bernstein WK, Khastgir T, et al. The use and clinical importance of a substrate-specific electrode for rapid determination of blood lactate concentrations. JAMA 1994;272:1678-85.

16 Ramakrishna B, Graham SM, Phiri A, et al. Lactate as a predictor of mortality in Malawian children with WHO-defined pneumonia. Arch Dis Child 2012;97:336-42.

17 Mandell LA, Wunderink RG, Anzueto A, et al. Infectious Diseases Society of America/American Thoracic Society consensus guidelines on the management of community-acquired pneumonia in adults. Clin Infect Dis 2007;44(Suppl 2):S27-72.

18 Trzeciak S, Dellinger RP, Chansky ME, et al. Serum lactate as a predictor of mortality in patients with infection. Intensive Care Med 2007;33:970-7.

19 Shapiro NI, Howell MD, Talmor D, et al. Serum lactate as a predictor of mortality in emergency department patients with infection. Ann Emerg Med 2005;45:524-8.

20 Wacharasint $\mathrm{P}$, Nakada $\mathrm{TA}, \mathrm{Boyd} \mathrm{JH}$, et al. Normal-range blood lactate concentration in septic shock is prognostic and predictive. Shock 2012;38:4-10.

21 Howell MD, Donnino M, Clardy P, et al. Occult hypoperfusion and mortality in patients with suspected infection. Intensive Care Med 2007;33:1892-9.

22 Juneja D, Singh O, Dang R. Admission hyperlactatemia: causes, incidence, and impact on outcome of patients admitted in a general medical intensive care unit. J Crit Care 2011;26:316-20.

23 Aslar AK, Kuzu MA, Elhan AH, et al. Admission lactate level and the APACHE II score are the most useful predictors of prognosis following torso trauma. Injury 2004;35:746-52.

24 Reed L, Carroll J, Cummings A, et al. Serum lactate as a screening tool and predictor of outcome in pediatric patients presenting to the emergency department with suspected infection. Pediatr Emerg Care 2013;29:787-91. 